\title{
NOTE ON A THEOREM OF BÔCHER AND KOEBE*
}

\author{
BY J. J. GERGEN
}

1. Introduction. In this paper a generalization of the following theorem, discovered independently by Bôcher $\dagger$ and Koebe, $\ddagger$ is established.

THEOREM 1. If $u(x, y)$ is continuous with its first partial derivatives in a plane region $R$, and if, for every circle $C$ contained in $R$,

$$
\int_{C} \frac{\partial u}{\partial n} d s=0
$$

where $n$ is the exterior normal to $C$, then $u$ is harmonic in $R$.

The generalization obtained is embodied in Theorem 2.

Theorem 2. If $v(x, y)$ is harmonic and positive in $R$, if $u(x, y)$ is continuous with its first partial derivatives in $R$, and if

$$
\int_{C} v \frac{\partial u}{\partial n} d s=\int_{C} u \frac{\partial v}{\partial n} d s
$$

for every circle $C$ contained in $R$, then $u$ is harmonic in $R$.

Taking $v$ as the constant one in Theorem 2, Theorem 1 is obtained.

Like Theorem $1, \S$ Theorem 2 has an analog in space, but,

* Presented to the Society, April 3, 1931.

$\dagger$ Bôcher, M., On harmonic functions in two dimensions, Proceedings of the American Academy of Arts and Sciences, vol. 41 (1906), pp. 577-583.

$\ddagger$ Koebe, P., Herleitung der partiellen Differentialgleichung der Potentialfunktion aus der Intergraleigenschaft, Sitzungsberichte der Berliner Mathematischen Gesellschaft, vol. 5 (1906), pp. 39-42.

$\S$ Koebe, loc. cit. For generalizations of Bôcher's and Koebe's Theorem of another type, see G. C. Evans, Fundamental points of potential theory, Rice Institute Pamphlets, vol. 7 (1920), pp. 252-329, espe ially.p. 286, and Note on a theorem of Bocher, American Journal of Mathematics, vol. 50 (1928), pp. 123-126; and G. E. Raynor, On the integro-differential equation of the Bôcher type in three space, this Bulletin, vol. 52 (1926), pp. 654-658. Evans, using the notion of the potential function of a gradient vector, shows that the conclusion of Theorem 1 holds with much lighter hypotheses both on $u$ and the character of the curves $C$. 
since no new essentially different details present themselves in the proof for space, we simply state this analog, and consider in detail only the plane case.

THEOREM 3. If $v(x, y, z)$ is harmonic and positive in a region $R$ in space, if $u(x, y, z)$ is continuous with its first partial derivatives in $R$, and if, for every sphere $C$ contained in $R$,

$$
\iint_{C} v \frac{\partial u}{\partial n} d s=\iint_{C} u \frac{\partial v}{\partial n} d s
$$

where $n$ is the exterior normal to $C$, then $u$ is harmonic in $R$.

The proof of Theorem 2 is elementary in character. The idea is to express $u v$ as a sum of integrals and deduce the character of $u$ from the properties of these integrals.

2. Proof of Theorem 2. We first observe that it is enough to prove the theorem in the case that $R$ is the interior of a circle $C$, and the hypotheses hold in the interior $R^{\prime}$ and on the boundary* of a circle $C^{\prime}$ concentric with $C$ but of larger radius. The problem, then, is to show that $u_{x x}$ and $u_{y y}$ exist and are continuous in $R$, and that

$$
\nabla^{2} u=u_{x x}+u_{y y}=0
$$

there.

Let $P(x, y)$ be any point in $R$. Let $\alpha^{\prime}$ be the radius of $C^{\prime}, \alpha$ the radius of $C$, and

$$
\rho=\frac{1}{2}\left(\alpha^{\prime}-\alpha\right) \text {. }
$$

Then, by (1), the hypothesis on $v$, and a classical formula, we have, for $0<t \leqq \rho$,

$$
\int_{C(P, i)} v \frac{\partial u}{\partial n} d s=\int_{C(P, t)} u \frac{\partial v}{\partial n} d s=\iint_{\sigma(P, t)} \phi d \sigma,
$$

where $\sigma(P, t)$ is the interior of the circle $C(P, t)$ of radius $t$ about $P$, and

$$
\phi(x, y)=\nabla u \cdot \nabla v=u_{x} v_{x}+u_{y} v_{y} .
$$

* That is to say, the hypotheses hold in a region containing $R^{\prime}$ and its boundary. 
It is the third integral in (3) that enables us to express $u v$ as a sum of integrals, whose properties lead to the conclusion of the theorem. Writing

$$
\sigma=\sigma(P, \rho), S^{\prime}=R^{\prime}+C^{\prime}, r=\left\{(x-\xi)^{2}+(y-\eta)^{2}\right\}^{1 / 2},
$$

we find that

$$
\begin{aligned}
\pi \rho^{2} u(x, y) v(x, y) & \\
= & \left\{\iint_{\sigma} u v d \sigma+\rho^{2}\left(\frac{1}{2}+\log \frac{1}{\rho}\right) \iint_{\sigma} \phi d \sigma\right. \\
& \left.-\frac{1}{2} \iint_{\sigma} \phi(\xi, \eta) r^{2} d \xi d \eta-\rho^{2} \iint_{S^{\prime}-\sigma} \phi \log r d \sigma\right\} \\
& +\rho^{2} \iint_{S^{\prime}} \phi \log r d \sigma \\
= & J^{\prime}(P)+J^{\prime \prime}(P), \text { say. }
\end{aligned}
$$

In fact, by (3),

$$
\begin{aligned}
\int_{0}^{\rho} \tau d \tau \int_{0}^{\tau} \frac{d t}{t} \int_{C(P, t)} v \frac{\partial u}{\partial n} d s & =\int_{0}^{\rho} \tau d \tau \int_{0}^{\tau} \frac{d t}{t} \int_{C(P, t)} u \frac{\partial v}{\partial n} d s \\
& =\int_{0}^{\rho} \tau d \tau \int_{0}^{\tau} \frac{d t}{t} \iint_{\sigma(P, t)} \phi d \sigma,
\end{aligned}
$$

or $K_{1}(P)=K_{2}(P)=K_{3}(P)$, say. Introducing, then, a system of polar coordinates $(r, \theta)$ with pole at $P$, we have

$$
\begin{aligned}
K_{1}(P) & =\int_{0}^{\rho} \tau d \tau \int_{0}^{2 \pi} d \theta \int_{0}^{\tau} v \frac{\partial u}{\partial r} d r \\
& =\int_{0}^{\rho} \tau d \tau \int_{0}^{2 \pi}\left\{u v-u(x, y) v(x, y)-\int_{0}^{r} u \frac{\partial v}{\partial r} d r\right\} d \theta \\
& =\iint_{\sigma} u v d \sigma-\pi \rho^{2} u(x, y) v(x, y)-K_{2}(P)
\end{aligned}
$$

so that

$$
\pi \rho^{2} u(x, y) v(x, y)=\iint_{\sigma} u v d \sigma-2 K_{3}(P)
$$


594

J. J. GERGEN

[August,

But, we see that

$$
\begin{aligned}
K_{3}(P) & =\int_{0}^{2 \pi} d \theta \int_{0}^{\rho} \tau d \tau \int_{0}^{\tau} \frac{d t}{t} \int_{0}^{t} r \phi d r \\
& =\int_{0}^{2 \pi} d \theta \int_{0}^{\rho}\left\{\frac{1}{2} \rho^{2}\left(\log \rho-\frac{1}{2}\right)+\frac{1}{4} r^{2}-\frac{1}{2} \rho^{2} \log r\right\} r \phi d r,
\end{aligned}
$$

upon changing the order of integration twice. Hence (4) follows.

Consider, now, the derivatives of $J^{\prime}$. We have

$$
\begin{aligned}
J^{\prime}(P)= & \int_{y-\rho}^{y+\rho} d \eta \int_{x-\psi}^{x+\psi} w d \xi+\left\{\int_{-\alpha^{\prime}}^{y-\rho} d \eta \int_{-\psi^{\prime}}^{\psi^{\prime}}+\int_{y-\rho}^{y+\rho} d \eta \int_{-\psi^{\prime}}^{x-\psi}\right. \\
& \left.+\int_{y-\rho}^{y+\rho} d \eta \int_{x+\psi}^{\psi^{\prime}}+\int_{y+\rho}^{\alpha^{\prime}} d \eta \int_{-\psi^{\prime}}^{\psi^{\prime}}\right\} w^{\prime} d \xi
\end{aligned}
$$

where

$$
\begin{aligned}
w & =w(x, y ; \xi, \eta)=u(\xi, \eta) v(\xi, \eta)+\phi(\xi, \eta)\left\{\frac{1}{2} \rho^{2}-\rho^{2} \log \rho-\frac{1}{2} r^{2}\right\} \\
w^{\prime} & =w^{\prime}(x, y ; \xi, \eta)=-\rho^{2} \phi(\xi, \eta) \log r \\
\psi & =\psi(\eta, y)=\left\{\rho^{2}-(\eta-y)^{2}\right\}^{1 / 2}, \psi^{\prime}=\psi^{\prime}(\eta)=\left(\alpha^{\prime 2}-\eta^{2}\right)^{1 / 2}
\end{aligned}
$$

We see, then, by using the fact that $u, v$ and $\phi$ are continuous in $S^{\prime}$, and the formula for differentiation under the integral sign, that, for $P$ in $R, J_{x}^{\prime}$ exists and is given by

$$
\begin{aligned}
J_{x}^{\prime}= & \int_{y-\rho}^{y+\rho}\{w(x, y ; x+\psi, \eta)-w(x, y ; x-\psi, \eta)\} d \eta \\
& +\int_{y-\rho}^{y+\rho}\left\{w^{\prime}(x, y ; x-\psi, \eta)-w^{\prime}(x, y ; x+\psi, \eta)\right\} d \eta \\
& +\iint_{\sigma} w_{x} d \sigma+\iint_{S^{\prime}-\sigma} w_{x}^{\prime} d \sigma \\
= & \int_{y-\rho}^{y+\rho}\{u(x+\psi, \eta) v(x+\psi, \eta)-u(x-\psi, \eta) v(x-\psi, \eta)\} d \eta \\
& -\iint_{\sigma}(x-\xi) \phi d \sigma-\rho^{2} \iint_{S^{\prime}-\sigma} \frac{(x-\xi)}{r^{2}} d \sigma ;
\end{aligned}
$$

which reduces to 
(5) $J_{x}^{\prime}=\iint_{\sigma}\left\{u v_{x}+v u_{x}-(x-\xi) \phi\right\} d \sigma-\rho^{2} \iint_{S^{\prime}-\sigma} \phi \frac{(x-\xi)}{r^{2}} d \sigma$, upon writing

$u(x+\psi, \eta) v(x+\psi, \eta)-u(x-\psi, \eta) v(x-\psi, \eta)$

$$
=\int_{x-\psi}^{x+\psi}\left\{u v_{x}+v u_{x}\right\} d \xi .
$$

From (5) and the continuity of $u$ and $v$ and their first partial derivatives, we deduce immediately that $J_{x x}^{\prime}$ and, as it is worth while noting for future purposes, $J_{x y}^{\prime}$ exist and are continuous in $R$. By analogy, $J_{y y}^{\prime}$ exists and is continuous in $R$.

Next, consider $J^{\prime \prime}$. The existence of $J_{x}^{\prime \prime}$ and $J_{y}^{\prime \prime}$ can be inferred from (4) and the existence of $J_{x}^{\prime}, J_{y}^{\prime}$ and the first partial derivatives of $u$ and $v$. We wish to know, further, that $J_{x x}^{\prime \prime}$ and $J_{y y}^{\prime \prime}$ exist and are continuous in $R$. To prove this, we first observe that $u, v^{-1}, J_{x}^{\prime}, J_{y}^{\prime}, J_{x}^{\prime \prime}, J_{y}^{\prime \prime}$ satisfy uniform Hölder conditions* in any closed domain $S^{\prime \prime}$ bounded by a circle $C^{\prime \prime}$ contained in $R$. The first four of these functions have this property because their first partial derivatives are continuous in $R$ and $R$ is convex $\dagger$ and contains $S^{\prime \prime}$, the last two because of a theorem of Dini. $\ddagger$ We next observe that from this property of $u, v^{-1}, \cdots$, $J_{y}^{\prime \prime}$, it follows that $\phi$ satisfies a uniform Hölder conditionin $S^{\prime \prime}$, for

$$
\phi=\left\{\nabla v \cdot \nabla J^{\prime}+\nabla v \cdot \nabla J^{\prime \prime}-\pi \rho^{2} u \nabla v \cdot \nabla v\right\} /\left(\pi \rho^{2} v\right),
$$

and thus $\phi$ is equal to a combination of sums and products of functions each of which satisfies a uniform Hölder condition in

* A function $f(P)$, defined on a set $E$, satisfies a uniform Hölder condition on $E$ if, $P$ and $Q$ being any two points of $E$,

$$
|f(P)-f(Q)|<A|P Q|^{\lambda}
$$

where $A$ and $\lambda$ are independent of $P$ and $Q$, and $\lambda>0$. Evidently, if $f_{1}(P)$ and $f_{2}(P)$ satisfy uniform Hölder conditions on $E, f_{1}+f_{2}$ and $f_{1} f_{2}$ have the same property.

$\dagger$ A region $R$ is convex if each segment, whose end points lie in $R$, lies in $R$.

$\ddagger$ U. Dini, Sur la méthode des approximations successives pour les équations aux dérivées partielles du deuxième ordre, Acta Mathematica, vol. 25 (1901), pp. 185-230. The function $J^{\prime \prime}$ is, of course, the potential function due to a distribution of continuous density $-\rho^{2} \phi$ over $S^{\prime}$. 
$S^{\prime \prime}$. The existence and continuity of $J_{x x}^{\prime \prime}$ and $J_{y y}^{\prime \prime}$ can now readily be deduced. We write

$$
\begin{aligned}
J^{\prime \prime} & =\rho^{2} \iint_{S^{\prime}-S^{\prime \prime}} \phi \log r d \sigma+\rho^{2} \iint_{S^{\prime \prime}} \phi \log r d \sigma \\
& =L^{\prime}+L^{\prime \prime}, \text { say. }
\end{aligned}
$$

Now $L_{x x}^{\prime}$ and $L_{y y}^{\prime}$ evidently exist and are continuous for $P$ in the interior of $S^{\prime \prime}$, while $L_{x x}^{\prime \prime}$ and $L_{y y}^{\prime \prime}$ exist and are continuous in $S^{\prime \prime}$ by a theorem of Hölder. ${ }^{*}$ Thus $J_{x x}^{\prime \prime}$ and $J_{y y}^{\prime \prime}$ exist and are continuous in the interior of $S^{\prime \prime}$. But $C^{\prime \prime}$ was arbitrary in $R$; and hence it follows that $J_{x x}{ }^{\prime \prime}$ and $J_{y y}^{\prime \prime}$ exist and are continuous in $R$.

The proof is now almost complete. Since $J_{x x}{ }^{\prime}, J_{y y}^{\prime}, J_{x x}{ }^{\prime \prime}, J_{y y}{ }^{\prime \prime}$ exist and are continuous in $R$, and since $v$ is positive and harmonic there, $u_{x x}$ and $u_{y y}$ exist and are continuous in $R$. It remains, then, only to prove that (2) holds. To prove this, we show that a contrary assumption leads to a contradiction. Suppose there is a point $P$ in $R$ at which $\left|\nabla^{2} u\right|=2 \beta$ is different from zero. Then we can choose $t$ so small that $\sigma(P, t)$ lies in $R$ and $\left|\nabla^{2} u\right|>\beta$ in $\sigma(P, t)$. Thus, since $v$ exceeds a positive constant $\epsilon$ in $\sigma(P, t)$ and $\nabla^{2} u$ is continuous there,

$$
\left|\iint_{\sigma(P, t)} v \nabla^{2} u d \sigma\right| \geqq \epsilon \beta \text { area } \sigma(P, t)>0 .
$$

But

(7) $\iint_{\sigma(P, t)} v \nabla^{2} u d \sigma=\int_{C(P, t)} v \frac{\partial u}{\partial n} d s-\int_{C(P, t)} u \frac{\partial v}{\partial n} d s=0$,

by the continuity of $\nabla^{2} u$, our hypotheses, and Green's formula. In (6) and (7) we reach the desired contradiction. The proof is now complete.

\section{HARVARD UNIVERSITY}

* Otto Hölder, Beiträge zur Potentialtheorie, Inaugural Dissertation, Stuttgart, 1882, p. 17. Hölder considers the second partial derivatives of a volume distribution. The same type of analysis, however, holds for plane distributions. 